\title{
CIGMA: aCtive Inventory service in Global e-MArket for enabling one-stop shopping over Internet shopping sites
}

\author{
Su Myeon Kim, Seungwoo Kang, Heung-Kyu Lee \\ Korea Advanced Institute of Science and Technology \\ EECS. Dept. \\ 373-1 Kusung-Dong, Yoosung-Ku, Taejeon, 305-701, Korea \\ \{smkim,swkang\}@nclab.kaist.ac.kr,hklee@casaturn.kaist.ac.kr \\ Junehwa Song \\ IBM T. J. Watson Research Center \\ P.O. Box 218, Yorktown Heights, N.Y. 10598 \\ junesong@us.ibm.com
}

\begin{abstract}
A fully connected Internet business environment will introduce a high level of dynamics to the business process. It may result in very frequent changes in business decision, and thus, information of various items may undergo constant changes. In addition, there could be a flood of similar shopping sites. In such a highly dynamic environment, ordinary online customers may feel that online shopping is not comfortable. Existing service models or systems cannot effectively reflect such a dynamic environment and support ordinary online customers. We propose a new E-commerce service called the CIGMA. The CIGMA provides catalog comparison and purchase mediation services over multiple shopping sites for ordinary online customers. The service is based on up-to-date information by reflecting the frequent changes in catalog information in real-time. It also matches the desire of the online customers for fast response since the service is provided based on the data cached in a high performance caching system. An important and challenging issue in realizing the CIGMA service is the design of merchant-side interface. This paper presents the CIGMA along with its merchant-side interface including service setup and deployment procedure.
\end{abstract}

\section{Introduction}

A fully connected Internet business environment will introduce a high level of dynamics to business process. Business processes can be promptly summarized and reported, and decisions can instantaneously be reflected. By effec- tively adapting to such a dynamic environment, merchants can increase their profits. However, such situation may result in very frequent changes in business decision. Thus, information on sales items may undergo constant changes. Unfortunately, to ordinary customers, this introduces a high degree of inconvenience in online shopping. An example can be seen from Cisco's case. By effectively adapting their business processes to the Internet environment, Cisco has shorten the term of their business closing to every six hours. Currently, they are trying to further shorten it to three hours. This means that they can accurately estimate the cost of their products, and hence change the sales conditions, every three hours. However, supposing that the sales conditions for popular goods are changed several times a day, customers may not be sure whether the conditions they saw at the merchant site ten minutes ago are correct. Thus, they can fall in a situation in which making shopping decision becomes very difficult.

Such inconvenience in online shopping is compounded by a flood of similar shopping sites on the Internet. For example, there are 7,513 registered sites in the Yahoo booksellers directory ${ }^{1}$. After narrowing the scope to the children's booksellers, there are still 298 sites. Online customers usually think that there are certainly other shopping sites with better sales conditions than the ones they have chosen and are not sure which site is the best one. This discomfort is much more serious than that of offline shopping due to the increased expectation on online shopping. In the conventional commerce environment, customers readily exclude visiting far-off shops considering the limits of time and distance; however, most online shopping sites have

\footnotetext{
${ }^{1}$ From Yahoo Web site(www.yahoo.com) on Mar. 14th, 2002
} 
nearly the same accessibility. To make matters worse, merchants contend with each other. Online merchants compete to provide better sales conditions than the ones provided by the others. In the end, such competition may cause a chain reaction among similar shopping sites within a short time $^{2}$. After all, online shopping in highly dynamic business environment can be described in a sentence:"NO ONE KNOWS HOW TO PURCHASE GOODS UNDER THE BEST SALES CONDITION".

To overcome such inconvenience in online shopping, customers need proper support. However, existing service models or systems cannot effectively reflect such a highly dynamic environment and support ordinary online customers. We propose a new EC service called "the aCtive Inventory service in Global e-MArket" (CIGMA)[15]. The CIGMA provides catalog comparison services over lots of shopping sites for ordinary online customers. A key characteristic of the CIGMA is that the service is provided, based on up-to-date information by reflecting frequent changes in catalog information in real-time. In addition, the service matches the desire of the online customers for fast response.

An important issue in realizing the CIGMA service is how to design the interface between the CIGMA and merchants. This interface includes detailed steps for service establishments and maintenance. It is a complicated problem since there exists a high degree of heterogeneity in different merchants and their systems. Efficiency is also an issue because the CIGMA, once in operation, easily becomes heavy-loaded dealing with numerous customers and merchants. In this paper, we describe the CIGMA focusing on its merchant-side interface including service setup and deployment procedures. Based on a Service Contract (SC) and a Merchant Wrapper(MW), the CIGMA provides a simple way of service setup and maintenance. Merchants only need to specify how to interact with the CIGMA in an SC, and once specified, the system automatically interprets and establishes services. Also, once in service, changes in data or system status are automatically catched and reflected to the CIGMA for continuous service.

Price comparison services [1, 2, 3] can partly address the on-line shopping problems arising from the situation where too many similar shopping sites co-exist. It gathers price information for an item from many shopping sites. Then, it provides customers with the comparison information. However, it can not guarantee the correctness of the comparison information because it generally updates the gathered price information periodically. So, the comparison information may not be up-to-date at the moment of access. In the end, customers need to visit the original sites to check the validity of the given prices. There also exists an approach based on instant gathering of price data [4]. This approach may

\footnotetext{
${ }^{2}$ Price War is the term used in economics to indicate such a chain reaction in price changes [12]
}

provide up-to-date price comparison information. However, it may incur a long delay that most online customers can not endure.

By keeping the catalogs to be up-to-date, the CIGMA effectively supports customers to shop online even in a highly dynamic e-commerce environment. Customers can easily choose the best sales conditions and do not have to undergo exhaustive surfing over lots of similar shopping sites. The CIGMA also provides purchase transaction mediation. By using this mechanism, online customers can buy goods from different shopping sites at the CIGMA site without visiting original shopping sites. We believe that customers can save time as well as money. For the merchants, the CIGMA provides a chance to increase their business profit. The CIGMA can be considered as a sales agency that sells merchants' items on behalf of them. With the help of the CIGMA's simple setup method, merchants can easily join the CIGMA service. In addition, most core operations, such as catalog update catch, notification, and format conversion, are automatized. So, merchants has little overhead in maintaining the CIGMA service.

This paper is organized as follows. In Section 2, the challenges of online shopping is illustrated by a motivating scenario. In Section 3, the design of the CIGMA is described. Section 4 discusses in detail the setup and deployment process in merchant-side. In Section 5, the implementation of the CIGMA prototype and sample shopping sites is described. Section 6 discusses related works. We conclude our work in Section 7.

\section{Motivating Scenario}

Consider two customers, Tom and Mary, who decide to buy a book, "Harry Potter and the Condition of Sales", at online bookstores.

There are numerous online bookstores on the Internet. Most of them sell the book since it is very popular. For instance, Amazon, Barnes\&Nobles and AllDirect ${ }^{3}$ sell the book at the price of $\$ 17.99, \$ 15.11$ and $\$ 15.59$, respectively. Suppose that at this time, Amazon decides to lower its price to $\$ 14.49$ to increase its market share. In a few minutes, Barnes\&Nobles also lowers its price to $\$ 14.39$ in accordance with its business policy: "Lower price than Amazon, Better service than all others". These events also force AllDirect to similarly change its selling price. In the end, this price change and competition cause a chain reaction in the online book market.

Now, one of the customers, Tom does not know much about e-commerce; he has only heard about the advantages

\footnotetext{
${ }^{3}$ The bookstores web sites are real. However, the characteristics of the sites mentioned in this paper are irrelevant to the actual characteristics of the sites. We use their names because they are popular. Other site names in this paper are used for the same purpose.
} 
of online shopping several times from his friends. He visits only the two well-known online bookstores: Amazon and Barnes\&Nobles. He finds out that Barnes\&Nobles has proposed a lower price than Amazon and he decides to purchase the book at Barnes\&Nobles. At that time, he realizes that there are a lot of other bookstores on the Internet and he begins to worry about whether or not his purchase at Barnes\&Nobles may be a good deal. He decides to visit more bookstores but realizing that there are so many, he randomly chooses several more bookstores and compares their prices for the book. In the end, he purchases the book from one of the bookstores he has visited recently. However, he feels that it is very uncomfortable to do shopping on the Internet. He feels that there must be a better sales condition elsewhere (and that he can find it if he has more time).

Mary, the other customer, as an experienced online shopper, knows that there are too many bookstores to visit and thus, visits MySimon, a popular web site with price comparison service for well-known bookstores. After several mouse clicks, she reaches the page showing a comparison table about the book and decides to buy it at AllDirect because of the lowest price. But at that moment, she notices a warning at the bottom of the web page which states: "Please note: PRICE shown here may NOT reflect the CURRENT price at the particular store. Be sure to CHECK both the price and the availability when viewing the product at the store". After reading this, she visits the AllDirect web site and finds out that the book is already sold out, and she is compelled to visit each bookstore (listed for comparison at MySimon) to verify the price and the availability. In the end, she is able to purchase the book at the lowest price but only after a long series of manual web search. Both Tom and Mary need to undergo an exhaustive web search that may be impractical and at many times, useless.

By visiting the CIGMA web site, both Tom and Mary can find a good bargain. For instance, if Tom were to visit the CIGMA and request the comparison information about the book he wants to buy, he will have known that there is a lot of bookstores selling the book. Then, he will have found the best sales condition among them. In addition, with the CIGMA mediating the purchase transaction, Tom will have purchased the book at the CIGMA website without having to access the bookstore web sites. Mary will have followed the same procedure. The remarkable difference between the CIGMA and the conventional price comparison service can be described in an advertising sign at the top of the CIGMA web page: "Please note: We always keep the catalog and comparison information to be UP-TODATE. NEVER waste time visiting each store web site to VERIFY the information provided ".

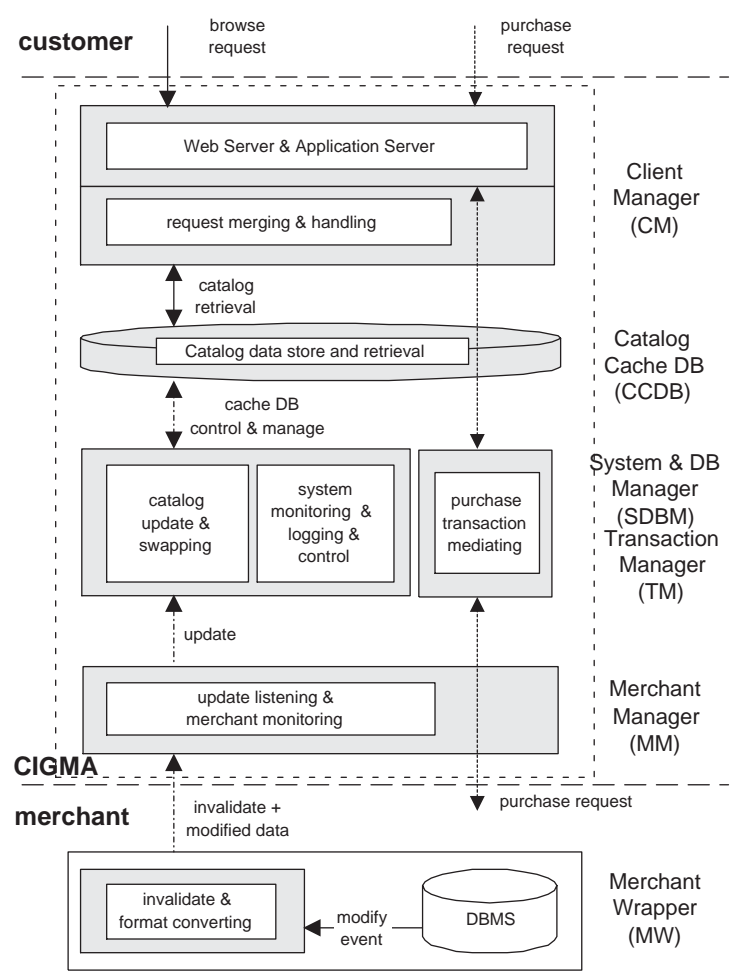

Figure 1. The CIGMA system architecture

\section{System Design}

\subsection{Overview}

The CIGMA has a modular structure. It consists of five server components and one remote component. The remote one, called Merchant Wrapper (MW), runs on each merchant server. The server components are Client Manager (CM), Merchant Manager (MM), Transaction Manager (TM), System \& DB Manager (SDBM) and Catalog Cache DB (CCDB). Figure 1 shows the CIGMA system along with a merchant's and a customer's. The function of each component will be described in Section 3.3.

We use main memory as a primary data storage to process a high rate of requests. Disks are used as a supporting medium to compensate the shortage of main memory space. We argue that persistency is less critical in the CIGMA since it deals with only the cached data which has replication in the original merchant servers. In addition, it has been reported that, in disk based caching systems, the overhead of disk accesses is a significant portion of overall load. Thus, in a busy system like the CIGMA with frequent requests and updates, the overhead of keeping data in disks will be even more significant. Our system trades off the lack of persistency with such an overhead. 


\subsection{Internal Service Flows}

There are three kinds of external service in the CIGMA: catalog browse, item purchase, and catalog update. The first two are for customers and the last, for merchants with each request being handled differently.

The catalog browse request is the most frequent one. It is first received by the Web server, which is a part of the CM. The CM then parses the request and constructs an equivalent DB query string. It sends the query string to the CCDB to retrieve the requested information such as a catalog comparison table, an entire catalog, an item category list, etc. Lastly, it replies to the request with an HTML page which is dynamically generated with the query result.

To handle a purchase request, a safety mechanism is required because it generally includes important information such as a credit card number, address, phone number, etc. To secure the purchase transaction processing, the CM communicates with customers using the secure HTTP protocol. Then, it forwards the received data to the TM. The TM also forwards the data to a proper merchant server at once. The result of the transaction is delivered from the merchant server to the customer in the opposite direction.

In the case of an update request, immediate processing is important. Each modification of source data at the merchant's DBMS is detected by the MW module. Then, the module constructs and sends an update request message to the $\mathrm{MM}$ at once. The MM forwards the message to the SDBM after verifying the integrity of the message. Then, the SDBM actually updates the cached data of the CCDB by composing a DB query string based on the message. After the CCDB update, the return value of the update operation (i.e., OK or NOT_OK) is forwarded to the MW in the reverse order.

\subsection{Major Components}

\subsubsection{Client Manager $(\mathrm{CM})$}

The CM takes charge of every communication with customers. It includes a Web server and a Web application server. The web application server is required to generate responses with dynamic contents. ${ }^{4}$ Via these servers occurs each interaction with customers. The CM receives both catalog browse requests and purchase requests from customers. As mentioned, the secure HTTP is used to handle purchase requests with security.

The $\mathrm{CM}$ also has a capability to merge identical requests from different customers. This improves the system performance when a lot of customers simultaneously send the same request for a popular item. For example, many people may send the comparison requests for a long awaited

\footnotetext{
${ }^{4}$ Popular web application servers are JAVA servlet, common gateway interface (CGI), active server page (ASP), etc.
}

book within a short period of time. The $\mathrm{CM}$ inspects the request queue and merges the same requests from different customers into a single internal request. With the help of request merging, the CIGMA can process a sudden rush of requests for popular goods.

\subsubsection{Merchant Manager (MM)}

The MM manages most of the interactions with merchants except those required for purchase transactions. Upon a catalog modification, the MM receives an update message from a merchant server. Then, it immediately notifies the SDBM of the event. There could be certain situations such as network partitioning, merchant server failure, network overload, etc., in which the MM cannot receive update messages. This may result in incorrect catalog information. To avoid this problem, the MM monitors messages from merchants to check the status of servers. If a merchant sends no messages during a predefined period, the MM notifies the SDBM of the situation. Then, the SDBM will initiate a suitable action. For example, the SDBM may invalidate all the catalogs from the unreachable server until the server responds again. A merchant is forced to send an empty message at the end of the predefined interval if there is no update.

\subsubsection{System \& DB Manager (SDBM)}

In addition to the cache DB update, the SDBM has two other functions : system monitoring and control, catalog swapping.

It monitors the status of the system and controls its components. For example, the SDBM monitors the frequency of the requests from customers and merchants and collects its statistics. It logs the statistics for later use. Based on the collected data, it controls major facilities of the components to improve their performance.

It also handles catalog swapping. When main memory is insufficient, the CIGMA may use disks as a secondary storage. It may swap in/out a portion of catalog data from/to disks. To select an optimal victim, the SDBM monitors the access and update frequency of each cached item. Based on these data, the SDBM selects a victim which is most likely to be unused for a long time. Since disk access is a very heavy task, the selection should be performed very carefully to reduce the number of disk accesses. After choosing a victim, the SDBM actually performs catalog swapping.

\subsubsection{Transaction manager (TM)}

The TM mediates purchase transactions. It does not process the transaction on behalf of merchants, but simply relays all the purchase-related information, i.e., order form, purchase order data, and transaction result, etc., between a customer 
and a merchant site. This approach is taken to avoid the complication and overhead which may be incurred by related business issues. The detailed procedure is omitted due to space constraint. (Refer to [15] for detailed discussions.)

The TM mediates purchase transactions in the order of time. An assurance for ordered processing is important when many requests arrive within a short period for a few popular goods with insufficient supply. A fair ordering is guaranteed by tagging messages with its original arrival time at the CM. A purchase request is processed according to the tag until the processing is completed.

\subsubsection{Catalog Cache DB (CCDB)}

The CCDB manages storing and retrieval of cached catalog data. Generally, the performance of DBMS is very critical to the overall performance of an e-commerce site. The CIGMA is expected to process a much higher number of update requests as well as customer requests than general shopping sites. Therefore, the performance of DBMS is even more critical in the CIGMA.

To support a very high number of retrievals and updates, we used main memory as the primary storage. Disks are used as a supporting medium to compensate the shortage of main memory space. We argue that persistency is less critical in the CIGMA since it deals with only the cached data that has replication in the original merchant servers. In addition, it has been reported that, in disk based caching systems, the overhead of disk accesses is a significant portion of overall load. Thus, in a busy system like the CIGMA with frequent requests and updates, the overhead of keeping data in disks will be even more significant. Our system trades off the lack of persistency with such an overhead.

\subsubsection{Merchant Wrapper (MW)}

The MW covers all the required works for a merchant to interact with the CIGMA. As shown in Figure 1, the MW is deployed on a merchant server for the merchant to join the CIGMA service. It catches modifications of catalog data in the merchant DB. Then, it sends the modification information to the CIGMA server. The detection of catalog change is automatically done using the DB trigger mechanism of the merchant DB. Trigger mechanisms are provided in most popular databases such as Oracle [5], DB2 [6], Sybase [7], MySq1 [8], PostgresSQL [9], etc.

Figure 2 shows the detailed update procedure and the structure fo the MW module. When an update event occurs at the merchant DB (1), a Trigger is automatically called by the merchant DBMS and the Trigger sends the modified data to the Event Reporter (2). The Event Reporter sends the modification information to the MW that is running on the merchant server (3). The message is received

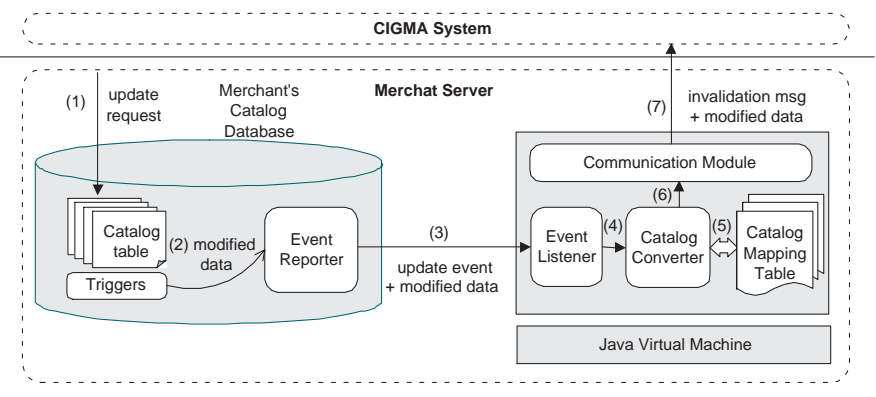

Figure 2. Merchant Wrapper architecture and update event propagation procedure at a merchant server

by the Event Listener module within the MW. Then, Cata$\log$ Converter converts the category as well as the format of the modification information if needed(4) referring Catalog Mapping Table ${ }^{5}(5)$. The Catalog Converter then composes an update message according to the SC (6). Lastly, the Communication Module sends the message to the CIGMA (7).

The MW also periodically sends out heart beat messages to the CIGMA, if there are no events during a predefined interval. Although the heart beat message carries no information, the message itself is important because it notifies the fact that the merchant server is alive. If there are neither modification messages nor heart beat messages, the CIGMA regards a merchant as having some critical problems. Thus, it may invalidate all catalog data from the merchant.

\section{Setup and Deployment of the CIGMA Ser- vice}

\subsection{Service Setup}

First, to join and start the CIGMA service, a merchant and the CIGMA should have clear understanding and agreement on the ways they interact with each other. For example, they should agree on the ways of communication, catalog classification, security and authentication, error correction, etc. This is not an easy task since each merchant may have very different system and business environments.

The CIGMA uses a Service Contract (SC) [14, 13] to ease the process of such agreement. An SC is a set of well defined rules represented in a machine readable form. It

\footnotetext{
${ }^{5}$ To cache heterogeneous databases into a unified one, it is also required for data in a original database to be converted into another form. The conversion rules are specified in Catalog Mapping Table. And, Catalog Converter do the conversion according to CMT. Interested reader can found the detail of content conversion in [15]
} 


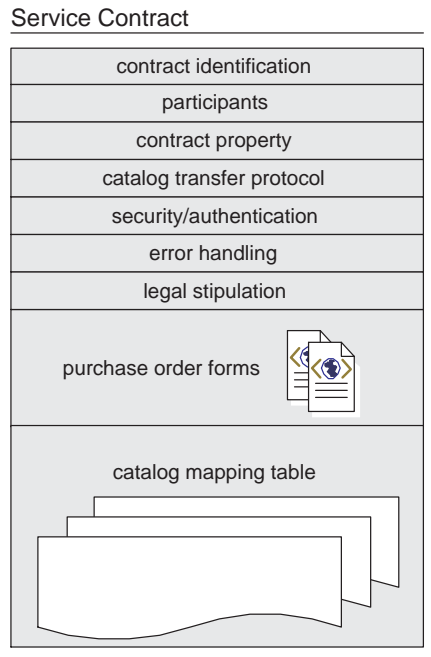

\section{Figure 3. Structure of the Service Contract}

is constructed and approved by a merchant as well as the CIGMA based on their agreement, and a constructed SC is kept by each party as a proof of agreement. Then, the $\mathrm{SC}$ is used to automate the process of system configuration on both parties. The signed SC is automatically fed into the systems. Then, each system component uses it as an input for the configuration. (The internal configuration procedure is described in Section 4.2.) The use of the SC also makes the re-configuration of the service to be done on the fly whenever either party wants to make some changes. They simply agree and sign on the modification of the SC and the new SC is fed to the systems again. The primary elements of a SC are shown in Figure 3.

Contract identification: The identification section assigns a unique identification (ID) to a contract.

Participants: The participants section provides information about participants. It consists of the parties' (a merchant and the CIGMA) names, their addresses, the names of persons responsible for the contract, telephone and fax numbers, etc.

Contract properties: Contract properties section includes the date and time when the contract is signed, start and end times of the service, contract cancellation conditions, etc.

Catalog Transfer Protocol: The catalog transfer protocol section has key attributes required to define interaction methods between a merchant and the CIGMA. The Supported actions field lists allowed operations (i.e., insert, update, retrieval, delete, etc.) between the two systems. The Communication channels field defines a network protocol and a network address for each supported action. It can be represented as a list, [action, protocol, source IP address, source port number, destination IP address, destination port number]. The protocol specifies a network transport protocol such as TCP or UDP. Lastly, the message structure field defines the message structure of each action. For instance, the structure of a delete message may be specified as a list, [delete, table id, primary key]. For simplicity of message parsing, the CIGMA provides several predefined message structures from which merchants can select for their own contract.

Security and authentication: The security and authentication section specifies the mechanism for protecting communications from malicious interrupts or attacks. There may be a number of combinations according to the given security options and mechanisms. To simplify the specification of this security rules, the CIGMA provides a few predefined levels. The CIGMA and a merchant negotiate with each other to choose a proper level. A simple example is as follows.

Level 1: [communication: DES, dynamic conf: disable] The highest security level: every communication is encrypted/decrypted based on the DES. The dynamic configuration of communication protocol is not allowed.

Level 2: [communication: DES, dynamic conf: enable] Every communication is secured by DES, but, it allows the dynamic re-configuration of communication protocol.

Level 3: [communication: plain, dynamic conf: disable] The dynamic re-configuration is not allowed: no encryption/decryption in communication.

Level 4: [communication: plain, dynamic conf: enable] The lowest security level: no encryption/decryption in communication. Dynamic re-configuration is allowed.

Error handling: The error handling section describes what-to-do upon errors. It may define the maximum number of retries and the corresponding action upon a communication time-out. It also defines the interval of heart beat messages.

Legal stipulation: Legal stipulation defines the legal aspects of the contract. For example, it may specify which party is responsible for the financial loss under an exceptional condition.

Purchase order forms: The purchase order forms section contains Web pages required to mediate purchase transactions. The CIGMA uses these HTML pages upon customers' purchase requests. It is also described in detail in [15]. 
Catalog mapping table: The catalog mapping table holds how-to-convert information when we move catalogs from merchant's original DB to the cache DB.

\subsection{Service Deployment}

For a merchant to actually join the CIGMA, the procedure for merchant-side service deployment follows the construction of an SC. We make the deployment process easy and simple by providing the MW module. As noted in Section 3.3.6, the MW covers all tasks required for a merchant to join the CIGMA service. Thus, a merchant does not need to implement and setup many modules such as communication module, catalog conversion module, category re-classification module, etc. It only needs to install the MW. Besides, the configuration of the MW is done automatically by the use of an SC. The only burden will be on the implementation and installation of triggers in the catalog database. We plan to further facilitate this process by providing templates and examples of the triggers for several popular databases.

The MW module runs on a JAVA virtual machine. Thus, it does not affect the reliability of the merchant server itself. Also, by using JAVA's powerful access control mechanism, merchants can make sure of the safety of the MW module by restricting the access of the MW module. For example, the MW module can be restricted to access only a merchant DB's predefined network port and the CIGMA's update listening network port. Lastly, the CIGMA may provide the merchant with the MW module in the form of source code instead of machine readable byte code. Thus, merchants may inspect the source code and verify whether the module is secure and reliable. The MW module may get the certification of the module from an authorized third party agency before distribution. This certification can be easily done since there is only one MW module, which is shared by different merchants. Thus, just one time certification will suffice.

We have said that, in an e-commerce environment, frequent changes are expected in catalog data such as price and inventory status, etc. However the same is expected in the business or system environments and policies of merchants. For example, a merchant may want to sell new items or change communication methods. In the CIGMA, the reconfiguration process is automated since the configuration is separated from the implementation by the use of an SC. A merchant and the CIGMA only need to feed a new SC again after updating it.

After the MW module is installed in a merchant server, the merchant and the CIGMA configures their systems based on an SC. Since the SC contains the specification for all the interaction rules, the configuration process is simplified. As noted earlier, the configuration is done by sim-

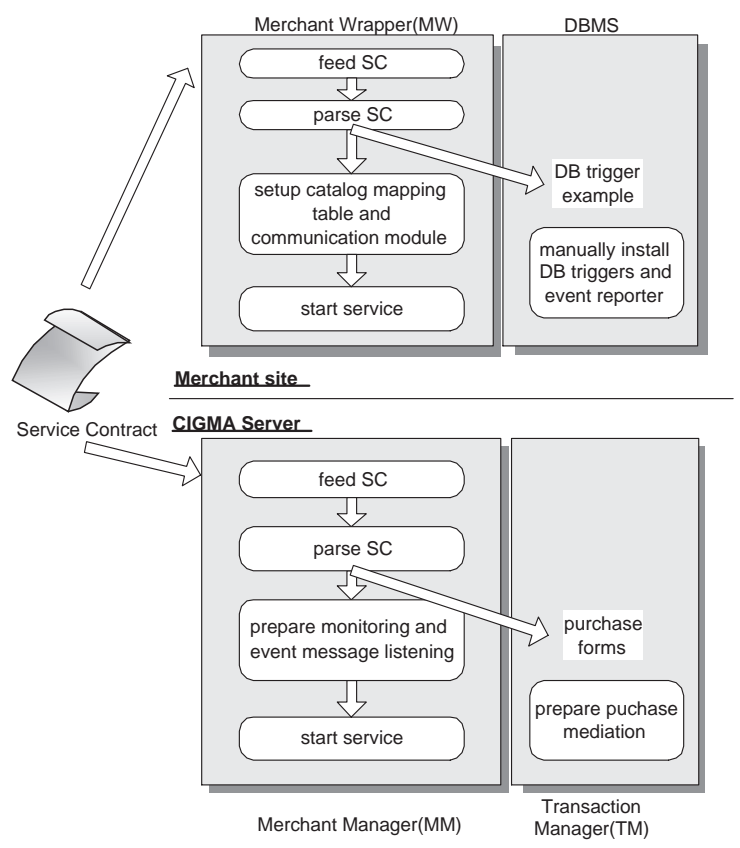

Figure 4. The configuration procedure at a new incoming SC

ply feeding the SC into the systems. The process is shown in Figure 4. The merchant, actually the MW module, first parses the SC and then sets up components such as the communication interfaces and the catalog mapping table.

The CIGMA also parses the SC. Then, it notifies related modules, i.e., MM's monitoring module and update listening module, etc., of the new service request. If needed, it also forwards configuration information, such as valid actions, protocols, addresses, etc., to each module by extracting the information from the SC. Based on this information, the modules prepare themselves for the new merchant. For example, the monitoring module adds the merchant server's network address and related meta data, such as heart beat message interval, to their watch list. After the internal setup is finished, the population function of the MM is executed. This function populates the CCDB by inserting catalogs of the newly incoming merchant. Then, the CIGMA becomes ready to serve the new merchant.

\section{Example Service}

We have built two sample shopping sites: Total Shop and Fragrance Online. Total Shop has a plan to become a general shopping mall and is trying to handle various items. Currently, Total Shop carries three kinds of items: MP3 players, perfumes, and computers. Figure 5 (a) shows a screen shot of Total Shop with the categories listed at 


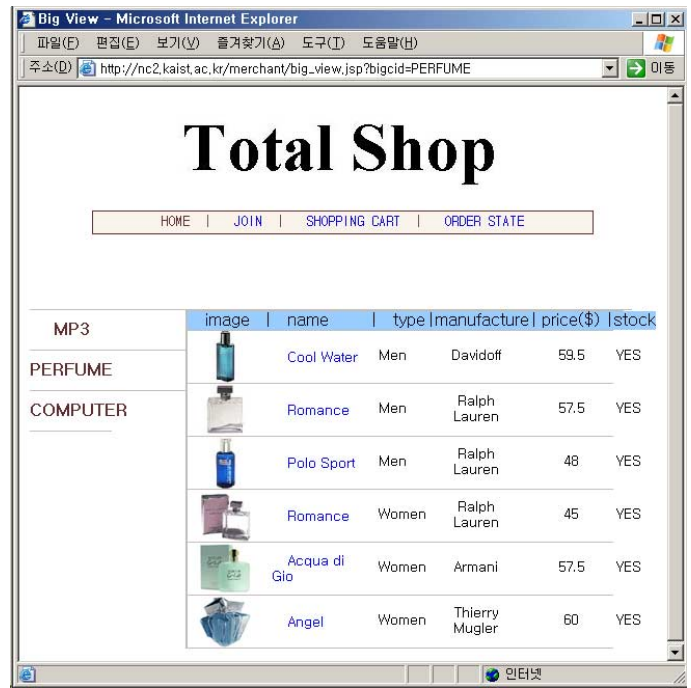

(a)

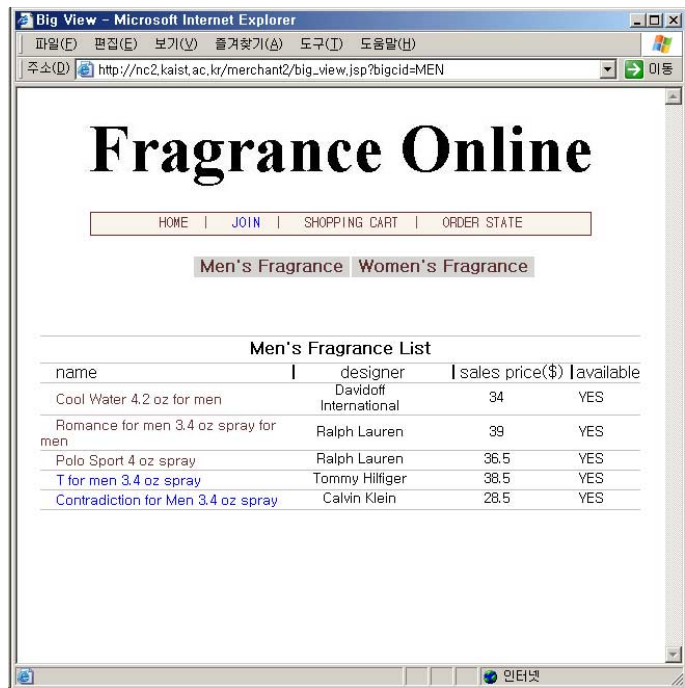

(b)

\section{Figure 5. The screen shots of two sample shopping sites - (a) Total Shop (b) Fragrance Online}

the left side of the screen. As shown in the figure, Total Shop has six kinds of perfumes. The fields of the perfume catalog are image_url, name, type, manufacture, price and stock. The type field indicates whether the item is for men or women.

Contrary to Total Shop, Fragrance Online specializes only in fragrances. As shown in Figure 5 (b), it has already classified the items into men's and women's fragrances. As such, the type field is not required in this shopping site's catalog. The figure shows the five catalogs of the men's fragrances.

Both shopping sites have joined the CIGMA service to increase their business profit. That is, they have already constructed their own SC's with the help of the CIGMA and installed MW modules and DB triggers. During the SC construction process, they have specified the category classification and catalog conversion information for each catalog.

The CIGMA is configured to handle various kinds of items. ${ }^{6}$ The items are classified into a two-level category: the first-level main category and the second-level subcategory. The leftmost frame in Figure 6 shows the main category. The next frame (in the middle) shows the subcategory that can be changed according to the main category. In the figure, the subcategory of the main category, "beauty", is shown. The CIGMA has its own catalog format as it has already contracted with both shopping sites. The rightmost

\footnotetext{
${ }^{6}$ The URL of the CIGMA is http://nc9.kaist.ac.kr/cigma/. The two sample merchant sites can be accessed via http://nc2.kaist.ac.kr/merchant/ and http://nc2.kaist.ac.kr/merchant2/.
}

frame in the figure shows the cached catalogs of perfumes from both shops.

The catalogs are automatically reclassified and converted according to the SC. The three catalogs of "PERFUME" with type "Men" in Total Shop are classified into the category "Perfumes for men", as are the five items in "Men's Fragrance" category of Fragrance Online. Note that the manufacture field of Total Shop is mapped into the vendor field of the CIGMA. The designer field of Fragrance Online is also mapped into vendor. Furthermore, the type field of Total Shop is not required in the CIGMA and it is dropped during the catalog conversion process. The catalog list in Figure 6 can be sorted according to each field of the comparison table. In addition, although we can not show here how the real-time update occurs, the catalog data of the CIGMA is updated immediately whenever the original data in the merchant DB is modified.

\section{Related Work}

Price comparison services $[1,2,3]$ is similar to our work. They provide catalogs for an item by gathering them from many merchant sites. However, they are not supported by an automated real-time update scheme. Many services update their data manually or use schemes based on periodic or aperiodic polling $[2,3,1]$. Compared to the CIGMA's case, we may say that those services help online customers in a best-effort style.

AddAll [4] provides price comparison service based on instant catalog searching and gathering upon customer's re- 


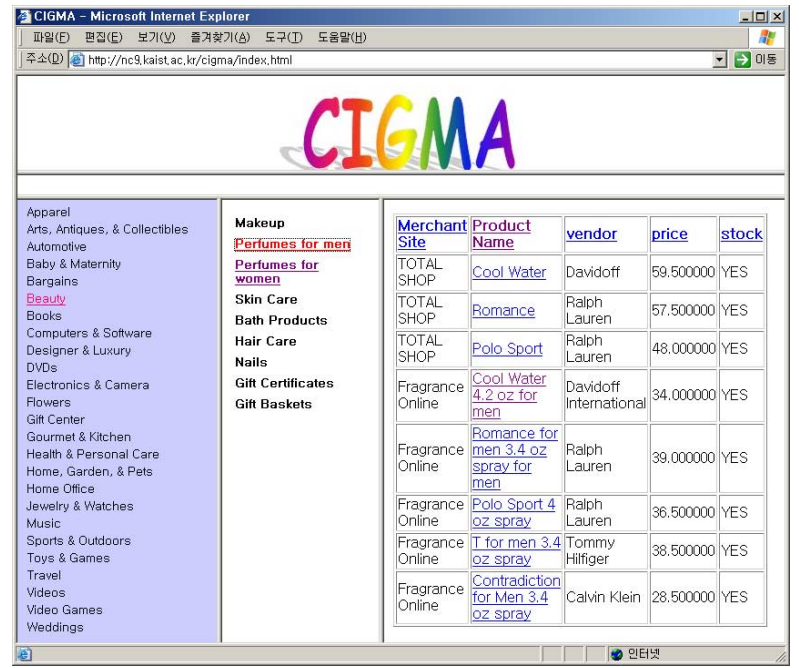

\section{Figure 6. The CIMGA's web page that displays} the catalog comparison table for perfumes.

quests. Thus, it can provide fresh information about an item. However, it also has a serious problem: the response time may be very long because it has to visit many shopping sites in an on-demand fashion. In addition, it issues multiple Web requests per each customer request and thus may cause a heavy traffic on the Internet.

These price comparison services are not a real shopping service. Neither of them have purchase transaction mediation functionality. Thus, customers have to visit the original shopping site to buy a selected item.

The B2B marketplaces $[10,11]$ intermediates between customers and merchants, and provides a set of services to support on-line purchasing. But, contrary to the CIGMA, most B2B marketplace model is designed and available only for business customers and/or transactions of a large volume. In addition, since B2B marketplaces do not deal with ordinary customers directly, performance concern is not serious in their design.

An electronic catalog mediating system has been proposed in [16]. Its goal is to mediate distributed catalog databases. It retrieves catalogs from multiple catalog DB. However, it does not cache catalogs but visit all catalog DB repeatedly upon customer's requests. Thus, this system can be used to provide instant search based price comparison service.

\section{Conclusions}

E-commerce (EC) has already become a major facilitator of business. It will become more important as the Internet grows rapidly. However, despite many advantages of the $\mathrm{EC}$, online shopping is an overloaded activity for most ordinary customers due to a highly dynamic e-commerce environment. For instance, they are not sure if a chosen sales condition is really a good one even after exhaustive Web surfing.

The CIGMA service can effectively support customers to do online shopping under a highly dynamic e-commerce environment. The CIGMA suggests the best sales conditions over multiple shopping sites and provides a convenient shopping environment for customers. Customers can save time and money by using the CIGMA service. For many merchants, the CIGMA helps them increase their business profit because the CIGMA is a sales agency. A challenge in realizing the CIGMA is in designing a merchant-side interface. We have described the CIGMA along with its merchant-side interface including service setup and deployment procedure.

\section{Acknowledgement}

This work was partly supported by the Korea Science and Engineering Foundation (KOSEF) through the Advanced Information Technology Research Center(AITrc).

\section{References}

[1] http://www.nextag.com/,NexTag - The Search Engine for Shoppers.

[2] http://www.mysimon.com/,mySimon - Compare products and prices from around the Web.

[3] http://www.dealtime.com/,DealTime - compare products, prices \& stores.

[4] http://www.addall.com/,AddALL - Book Search and Price Comparison

[5] http://otn.oracle.com/doc/oracle8i_816/appdev.816/a76939/ adg13trg.htm\#376, Oracle8i Application Developer's Guide - Fundamentals Release 2 (8.1.6).

[6] http://www-4.ibm.com/software/data/db2/udb/ad/v7/adg/db 2a0/frame3.htm\#trigger, DB2 Application Development Guide: Using Triggers in an Actie DBMS.

[7] http://my.sybase.com/detail?id=1355,Sybase Manual: Triggers.

[8] http://www.mysql.com/doc/A/N/ANSI_diff_Triggers.html, MySQL manual: 1.7.4.4. Stored Procedures and Triggers.

[9] http://www.postgresql.org/idocs/index.php?plpgsqltrigger.html,PostgreSQL 7.1 Documentation: Chapter 24. PL/pqSQL-SQL Procedural Language.

[10] http://service.ariba.com/, Ariba Supplier and Buyer Network.

[11] http://www.biz2biz.com/Marketplace/, Biz2Biz B2B Marketplace.

[12] L. M. B. Cabral. Introduction to Industrial Organization . MIT Press, 2001.

[13] A. Dan, T. Nguyen, M. Sachs, H. Shaikh, R. King, and S. Duri. The Coyote Project: Framework for Multi-party E-Commerce. LNCS 1513, pages 873-889, 1998. 
[14] A. Dan and F. Parr. The Coyote approach for Network Centric Service Applications: Conversational Service Transactions, a Monitor and an Applicat. In High Performance Transaction Processing (HPTS) Workshop, September 1997.

[15] S. M. Kim, S. Kang, H.-K. Lee, and J. Song. CIGMA: aCtive Inventory service in Global e-MArket for enabling onestop shopping over Internet shopping sites. Technical Report CS-TR-2002-178, Korea Advanced Institute of Science and Technology (KAIST), 2002.

[16] D.-M. Lincke and B. Schmid. Mediating Electronic Product Catalogs. Communication of the ACM, 41(7):86-88, July 1998. 